\title{
20th Workshop of the International Stroke Genetics Consortium, November 3-4, 2016, Milan, Italy
}

\author{
2017;3:e136; doi: 10.1212/NXG.0000000000000136
}

\subsection{6 \\ ISGC research priorities Daniel Woo, $M D^{l}$; Stephanie Debette, $M D^{2}$; Christopher Anderson, $M D^{3}$}

${ }^{1}$ University of Cincinnati, OH; and ${ }^{2}$ University of Bordeaux, France; ${ }^{3}$ Massachusetts General Hospital, Boston.

OBJECTIVE: To develop a research priority list for the International Stroke Genetics Consortium (ISGC). BACKGROUND: Previously, stroke progress review groups and other activities provided an opportunity for members within the field to develop priorities for research. These filtered into larger categories which then published the top research priorities of their given field. However, these review groups are no longer forming and thus there has been no clear direction on what the research priorities and needs for stroke genetics research. DESIGN/METHODS: We polled members of the stroke genetics community from around the world including collaborations of the major stroke genetics research efforts currently under way asking each respondent to provide their top 3 research priorities. Each priority was then sorted by the authors into major categories based upon the type of suggestion made. The results were presented for commentary and feedback during the presentation. RESULTS: Deeper Phenotyping related topics were presented in $84 \%$ of respondent top research priorities. These included topics related to ethnic/racial variation, third world countries, deeper evaluation of subtypes of stroke. Translational science related topics were present in $40 \%$ of respondent top research priorities and consisted of translating our current findings into a greater understanding of their relationship to stroke and clinical pathophysiology. Pooling of our resources through data banking and sample banking was a topic in $36 \%$ of respondents. Recovery/outcome related topics were suggested in $32 \%$ of respondents and utilizing novel techniques such as epigenetics, whole genome sequencing, proteomics and metabolomics were present in $28 \%$ of respondents. Other topics were not present in more than $25 \%$ of the responses for top research priorities. CONCLUSIONS To summarize, greater sample size and research within subtypes and endophenotypes of stroke was the top research priority of the ISGC. In addition, translating our existing findings, pooling our resources, recovery/outcomes research and utilization of novel techniques were among the top research priorities of the ISGC. A working group will be assembled to draft the ISGC Research Priorities paper.

Disclosure: None.

\subsection{7}

\section{Genetics of intracerebral hemorrhage: Progress}

report Daniel Woo, $M D^{1}$; Christopher Anderson, $M D^{2}$, Jonathan Rosand, $M D^{2}$

${ }^{1}$ University of Cincinnati, $\mathrm{OH}$; and ${ }^{2}$ Massachusetts General Hospital, Boston.

OBJECTIVE: To review the progress of current ISGC Intracerebral Hemorrhage (ICH) Genetics grants. BACKGROUND: The GOCHA (Rosand PI) and GERFHS (Woo PI) studies were separately funded efforts to recruit ICH cases and perform genome wide association genotyping. Along with collaboration through the ISGC, meta-analysis and replication identified an association with a region of Chrlq22 locus with non-lobar ICH. DESIGN/METHODS: GERFHS-IV (Woo and Rosand-multiple PI) has been recently funded to recruit 1,000 cases of ICH of which 500 non-lobar ICH will undergo deep sequencing of the previously identified locus along with RNA sequencing. One of the genes in the region is a master regulator of a cascade of genes and other genes in the area may be the true cause of association. RNA Sequencing will permit evaluation of alternative gene-splicing transcripts, gene fusion, gene expression changes, post-translational modifications, small RNA, miRNA and non-coding RNA sequencing. Cases are identified through hot-pursuit/active prospective recruitment. All centers were IRB approved as of September with 39 cases recruited by late October. RESULTS: The Ethnic/Racial Variations of Intracerebral Hemorrhage (ERICH) study was funded to and successfully recruited 3,000 cases of ICH (1/3 black, white and Hispanic) and have now completed 2,907 race/ethnic/demographically matched controls of the 3,000. Numerous papers have been written from these grants and proposals which were reviewed. The ERICH-L study (Rosand and Woo-Multiple PI) was recently funded to perform follow-up of the cases for recurrence, mortality, and cognitive outcomes assessment. Achieved IRB approval in September 6, 2016 and have since made 3,449 calls, 135 enrollments of which 40 agreed to enroll for a total of 175 cases. Have contacted $17.5 \%$ of the total cohort of which 175 agreed to enroll which extrapolates to 875 of the 900 cases we hope to enroll. ICH Recovery grant was scored but not funded and will be resubmitted. The proposal will follow-up on 500 cases of ICH from the parent GERFHS study above and perform 3T diffusion tensor imaging, T1W 3D imaging, susceptibility weighted imaging and 3- and 12-month followups to include a detailed evaluation of motor, cognitive, activity and urogenital distress. CONCLUSIONS: The ISGC ICH study group is pursuing deep sequencing of the Chr1q22 locus along with RNA sequencing. We anticipate completing the multi-ethnic case-control cohort and will seek funding and resources to perform a multi-ethnic genome sequencing or GWAS study. Will follow-up this cohort for recurrence and major outcome measures and pursuing additional research to gather detailed information on outcomes after ICH.

Study Supported By: NS59727, NS36695, NS69763.

Disclosure: None.

\subsection{8}

DNA-methylation and aging: Contribution of biological age to mortality after ischemic stroke Carolina Soriano-Tárraga, PhD; Marina Mola-Caminal, MSc; Eva Giralt-Steinhauer, MD, PhD; Rosa M. Vivanco-Hidalgo, MD; Angel Ois, MD, PhD; Ana Rodríguez-Campello, MD;
Elisa Cuadrado-Godia, MD, PhD; Jaume Roquer, MD, PhD; Jordi Jiménez-Conde, $M D, P h D$

Department of Neurology, Hospital del Mar; Neurovascular Research Group, IMIM (Institut Hospital del Mar d'Investigacions Mèdiques); and Universitat Autònoma de Barcelona/DCEXS-Universitat Pompeu Fabra, Barcelona, Spain.

OBJECTIVE: We seek to analyze the impact of biological age in mortality after an ischemic stroke. BACKGROUND: Stroke has a great impact in functional status of patients, although there are substantial interindividual differences in recovery capacity. Apart from stroke severity, age is considered an important outcome predictor after stroke, but aging is not only due to chronological age. There are age-related DNA-methylation changes in multiple CpG sites across the genome that can be used to estimate the biological age (b-Age). DESIGN/ METHODS: We include 600 individuals with acute ischemic stroke assessed in Hospital del Mar (Barcelona). Demographic and clinical data such as chronological age (c-Age), vascular risk factors, NIHSS at admission, recanalization treatment (rtPA or endovascular treatment), previous modified Rankin scale ( $\mathrm{p}-\mathrm{mRS}$ ) and $\mathrm{mRS}$ at 3 months were registered. Biological age (b-Age) was estimated with Hannum algorithm, based on DNA methylation in 71 CpGs. RESULTS: The bivariate analyses for association with mortality showed significant results of NIHSS, c-Age, b-Age, p-mRS, current smoking, sex and atrial fibrillation (all with $p<0.001$ ), and recanalization treatment, dyslipidemia and coronary heart disease (all with $p \leq 0.05$ ). In multivariate models, b-Age kept its significance $(p=0.024)$ nullifying c-Age $(p=0.81)$. Initial NIHSS, p-mRS and atrial fibrillation kept also significant results. Stratifying by age quartiles, young strokes $(<69$ years, $\mathrm{N}=158$ ) showed to have a better mortality prediction with b-Age $(\mathrm{AUC}=0.78)$ than with $\mathrm{c}-\mathrm{Age}(\mathrm{AUC}=0.70)$, close to significance $(p=0.125)$. This difference was not observed in older IS. In young strokes, the multivariate model including b-Age and adjusted by NIHSS and p-mRS had a good predictive value of AUC $=0.82$, better than a model including c-Age instead b-Age, that showed an AUC $=0.79(p=0.052)$. In older strokes there were no differences between models. CONCLUSIONS: In conclusion, biological Age, estimated by DNA methylation, is an independent predictor of stroke mortality, irrespective to chronological age, with pronounced impact in young ischemic stroke similar to NIHSS "Healthy aging" affects the outcome after an ischemic stroke.

Study Supported By: Agència de Gestió Ajuts Universitaris de Recerca (2014 SGR 1213); Spain's Ministry of Health (Ministerio de Sanidad y Consumo) through the Carlos III Health Institute (ISCIII-FIS-FEDER-ERDF, PI12/01238, PI15/00451, PI15/00445); RETIC (RD12/0042/0020, RD12/0042/0013); RecerCaixa 2013 research grant (JJ086116).

Disclosure: None.

\subsection{9}

The challenge of CADASIL diagnosis: The value of the clinical phenotype Anna Bersano, $M D, P h D^{t}$; Gloria Bedini, $\mathrm{PhD}^{t}$; Paolo Vital, $M D^{2}$; Greta Brenna, PhD ${ }^{1}$; Hugh Stephen Markus, Prof'; Silvia Lanfranconi, $M D^{4}$; Giuseppe Micieli, $M D^{2}$; Franco Taroni, $M D^{t}$; Cinzia Gellera, $M D^{t}$; Silvana Penco, $P h D^{5}$; Paola Carrera PhD ${ }^{6}$; Giorgio Battista Boncoraglio, $\mathrm{MD}^{I}$; Eugenio Parati, $\mathrm{MD}^{I}$; Maria Luisa De Lodovici, MD'; Giorgio Bono, Prof; Francesca Mazucchelli, MD'; Davide Zarcone, $M D^{8}$; Maria Vittoria Callone, $M D^{9}$; Patrizia Perrone, $M D^{9}$; Bianca Maria Bordo, $M D^{I 0}$; Alessandro Pezzini, Prof ${ }^{1}$; Alessandro Padovani, Prof ${ }^{1}$; Anna Cavallini, $M D^{2}$; Simone Beretta, $\mathrm{MD}^{12}$; Carlo Ferrarese, Prof ${ }^{2}$; Cristina Motto, $\mathrm{MD}^{5}$; Elio Agostoni, $M D^{5}$, Graziella Molini, $M D^{13}$; Francesco Sasanelli, $M D^{13}$; Manuel Corato, $M D^{14}$; Simona Marcheselli, MD ${ }^{14}$; Maria Sessa, MD ${ }^{6}$; Giancarlo Comi, Prof $f^{6,7}$; Nicoletta Checcarelli, $M D^{15}$; Mario Guidotti, $M D^{15}$; Davide Uccellini, $M D^{16}$, Lucia Tancredi, $M D^{17}$; Marco Arnaboldi, $M D^{17}$; Barbara Incorvaia, $M D^{18}$; Carlo Sebastiano Tadeo, $M D^{18}$; Laura Fusi, $M D^{19}$; Giampiero Grampa, $M D^{19}$; Cristina Cereda, $M D^{2}$; Caspar Grond-Ginsbach, $P h D^{20}$; Livia Candelise, Prof; On behalf of Lombardia GENS-group

${ }^{1}$ IRCCS Foundation C, Besta Neurological Institute-Milan, Italy; ${ }^{2}$ IRCCS Foundation C, Mondino Neurological Institute-Pavia, Italy; ${ }^{3}$ University of Cambridge, UK; ${ }^{4}$ Maggiore Policlinico Hospital Foundation IRCCS Ca' Granda-Milan; ${ }^{5} \mathrm{Niguarda} \mathrm{Ca}$ ' Granda Hospital, Milano, Italy; 'IRCCS Ospedale San Raffaele, Milano, Italy; ${ }^{7}$ Circolo Hospital and Macchi Foundation, Varese Hospital Varese, Italy; ${ }^{8} \mathrm{~S}$. Antonio Abate Hospital-Gallarate, Italy; ' ${ }^{9}$ Legnano and Cuggiono Hospital, Italy; ${ }^{10}$ Ospedale di Desio, Italy; ${ }^{11}$ Spedali Civili, University of Brescia, Italy; ${ }^{12}$ San Gerardo Hospital, Milan Center for Neuroscience, University of Civili, University of Brescia, Italy; ${ }^{12}$ San Gerardo Hospital, Milan Center for Neuroscience, University of
Milano-Bicocca, Italy; ${ }^{13} \mathrm{AO}$ Melegnano-Ospedale di Vizzolo Predabissi, Melegnano, Italy; ${ }^{4}$ Istituto Clinico Humanitas, Rozzano, Italy; ${ }^{15}$ Valduce Hospital, Como, Italy; ${ }^{16}$ Tradate Hospital-Tradate, Italy; ${ }^{17}$ Sant'Anna Hospital, Como, Italy; ${ }^{18}$ Istituto Clinico Città Studi, Milan, Italy; ${ }^{19}$ Ospedale di Circolo, Saronno, Italy; and ${ }^{20}$ Heidelberg University Hospital, Germany.

OBJECTIVE: The aims of our study are (1) to describe the clinical and neuroradiologic features of a well phenotyped Italian population (belonging to the Lombardia GENS study) of NOTCH3positive and negative patients, searching for possible disease specific markers, and (2) to assess sensibility and specificity of the already presented CADASIL scale and the accuracy of a new possible diagnostic algorithm. BACKGROUND: Cerebral autosomal dominant arteriopathy with subcortical infarcts and leukoencephalopathy (CADASIL) is the most common inherited small vessel disease, caused by mutations of $\mathrm{NOTCH} 3$ gene. The phenotype heterogeneity and the lack of specific clinical and neuroimaging findings make challenging the identification of patients to be addressed to genetic screening. Guidance on type of patients in whom there is a yield sufficient to merit testing is important, since $\mathrm{NOTCH} 3$ genetic screening is costly and time consuming. Pre-genetic diagnostic criteria have been previously proposed, including a specific CADASIL scale, suggesting a 14 point score cutoff as suggestive of CADASIL. However, these criteria were derived from heterogeneous population and not completely validated. DESIGN/METHODS: One hundred two patients (mean age $55+$ 12 years) with ischemic or hemorrhagic stroke or TIA, undergone genetic test for CADASIL, were included in the present study. For each patient detailed demographic and clinical data were collected and MRI images were centrally analyzed for the presence of lacunar infarcts, 
microbleeds, temporal lobe involvement, global atrophy and leukoaraiosis (Fazekas scale). Fisher exact test was applied to calculate association between genetic results and algorithm variables whereas ROC curve was used to find the best algorithm cut-off predicting genetic results. RESULTS: We detected disease-related mutations in $9 \%$ of our suspected cases. Familia history for stroke $(p=0.011)$, thalamic lacunes $(p=0.033)$, external capsule lesions $(p=$ $0.029)$ and severe leukoaraiosis (Fazekas $=3)(p=0.05)$ were significantly associated with the genetic diagnosis of CADASIL. Sensibility (SE) and specificity (SP) of the published CADASIL scale were respectively 0.778 and 0.771 , whereas $\mathrm{SE}$ and $\mathrm{SP}$ of our algorithm were 0.778 and 0.699. CONCLUSIONS: Familial history for stroke and neuroimaging data are the most important features to distinguish NOTCH3 positive from negative patients. Our algorithm demonstrated to have an accuracy comparable with the published CADASIL scale.

Study Supported By: The Lombardia GENS (GENetics of Stroke) project has received funding from the Regione Lombardia Government as a Research Independent Project (DGR no. VIII/006128-12/12/2007). Hugh Markus is supported by a National Institute for Health Research (NIHR) Senior Investigator award and his work is supported by the NIHR Cambridge University Hospitals Comprehensive Biomedical Research Centre (BRC).

Disclosure: None. Lombardia GENS is an investigator-driven, academic, non-profit consortium and is publicly funded.

\subsection{0}

\section{A genome-wide association study identifies novel} susceptibility variants for small-vessel ischemic stroke in a Han Chinese population Tai-Ming Ko, PhD', Tsong-Hai Lee, MD, $\mathrm{PhD}^{2}$; Chien-Hsiun Chen, $\mathrm{PhD}^{\prime}$; Yuan-Tsong Chen, $M D$, PhD ${ }^{I}$; Jer-Yuarn Wu, PhD

${ }^{1}$ Institute of Biomedical Sciences, Academia Sinica, Taipei, Taiwan; and ${ }^{2}$ Chang Gung Memorial Hospital, Linkou Medical Center, and Chang Gung University College of Medicine, Taoyuan, Taiwan.

OBJECTIVE: We sought to identify genetic factors that contribute to small-vessel occlusion (SVO) stroke in 2 independent samples of Han Chinese individuals. BACKGROUND: Previous studies have found genetic associations in cardioembolic and large-vessel ischemic stroke. However, no robust genetic association has been reported for SVO ischemic stroke in a Han Chinese population. DESIGN/METHODS: A genome-wide association study (GWAS) was conducted on 342 individuals with SVO stroke and 1,731 controls from a Han Chinese population residing in Taiwan. The study was replicated in an independent Han Chinese population comprising an additional 188 SVO stroke cases and 1,265 controls. RESULTS The mean age of the SVO group in the discovery group and replication group was 57.9 years old and 56.0 years old, respectively. Three SNPs clustered at chromosome 3 , with $p$ values between $2.52 \times 10^{-6}$ and $3.59 \times 10^{-6}$, were identified. Other 5 loci at chromosome 3, 4, 6, and 14 were also identified in the independent Han Chinese population. CONCLUSIONS: To our knowledge, this is the first GWAS for Asian single cohort of SVO stroke conducted in a Han Chinese population. The understanding of the genetic basis of SVO could be valuable in the development of treatments for specific subtypes of ischemic stroke.

Study Supported By: Academia Sinica Genomic Medicine Multicenter Study, Taiwan (4005-GMM) and Chang Gung Memorial Hospital (BMRP 274, CMRPG35072, CMRPG 35073, and CMRPG 39082).

Disclosure: None.

\subsection{1}

The Brazilian Institute of Neuroscience and Neurotechnology (BRAINN): A center of excellence for research, technology development and care for patients with epilepsy and stroke Fernando Cendes, $M D, P h D^{\prime}$, Iscia Lopes-Cendes, $\mathrm{MD}, \mathrm{PhD}^{2}$; On behalf of the BRAINN

${ }^{1}$ Department of Neurology, and ${ }^{2}$ Department of Medical Genetics, School of Medical Sciences, University of Campinas-UNICAMP, SP, Brazil.

OBJECTIVE: To present the goals and some preliminary results of the BRAINN. BACKGROUND: The aim of the BRAINN is the investigation of basic mechanisms that lead to epilepsy and stroke and the injury mechanisms that follow the disease onset and progression. DESIGN/METHODS: The ensemble of sub-projects in BRAINN spans from the biological and genetic aspects of epilepsy and stroke, through imaging and clinical aspects, rehabilitation and dissemination of knowledge. These sub-projects include association studies and next-generation sequencing to identify candidate loci and genes related to predisposition and severity of stroke; the development of rehabilitation systems; investigation of the relationship between the default mode network (DMN) structural and functional connectivity (FC) with vascular cognitive impairment in stroke patients; evaluation of subtle white and gray matter abnormalities associated with asymptomatic carotid stenosis; among others. RESULTS: Some of the initial results include: (1) the identification 314 Copy Number Variations (CNVs) present among hemorrhagic stroke patients from Joinville, Brazil. We are currently evaluating whether these CNVs are also present in different populations as part of the ISGC. (2) Stroke patients with depression and/or anxiety showed an increased DMN FC in left insula, left inferior parietal gyrus, right medial frontal gyrus and left basal nuclei, when compared to stroke controls. Specific correlation between clinical scores and DMN FC indicated that depression symptoms correlated with left inferior parietal gyrus, while anxiety symptoms correlated with cerebellum, brainstem, basal nuclei and right middle frontal gyrus. (3) We observed significant macro- and microstructural white matter abnormalities in 25 patients with asymptomatic $\geq 70 \%$ /occlusion carotid stenosis compared to 25 healthy controls, evaluated with voxelbased morphometry (VMB) and diffusion tensor images (DTI). While DTI findings were diffuse and symmetrical in both hemispheres, VBM gray matter atrophy occurred in areas corresponding to the anterior circulation ipsilateral to the carotid stenosis. CONCLUSIONS: The mission of BRAINN is to develop new methods and techniques to improve the understanding of mechanisms of damage, plasticity and repair in epilepsy and stroke; and to apply these results to improve diagnosis, prevention, rehabilitation and treatment.

Study Supported By: Fapesp Grant \#2013/07559-3.

Disclosure: None.
2016.042

Departure from Hardy-Weinberg Equilibrium within

common deletions Bowang Chen $^{\prime}$; Michal Korostynski ${ }^{2}$; Agnieszka Stowik ${ }^{3}$; John W. Cole ${ }^{4}$; Arne Lindgren ${ }^{5}$; Caspar Grond-Ginsbach ${ }^{6}$

${ }^{1}$ Department of Biology, South University of Science and Technology of China, Shenzhen, China; ${ }^{2}$ Department of Molecular Neuropharmacology Institute of Pharmacology PAS, Krakow, Poland; ${ }^{3}$ Department of Neurology, Jagiellonian University Krakow, Poland; ${ }^{4}$ Department of Neurology, Veterans Affairs Medical Center and Department of Neurology University of Maryland School of Medicine, Baltimore; ${ }^{5}$ Department of Clinical Sciences Lund, Neurology, Lund University and Department of Neurology, Skåne University Hospital, Sweden; and ${ }^{6}$ Department of Neurology, University of Heidelberg, Germany.

OBJECTIVE: To use CNV analysis to examine if Hardy Weinberg Equilibrium is altered in common deletion areas of the genome. BACKGROUND: Genetic variants located within a common genomic deletion are less likely to be heterozygous than variants in regions with normal copy number. We hypothesize that Hardy Weinberg Equilibrium (HWE) - violating SNPs cluster in regions with common deletion. DESIGN/METHODS: SNP-genotypes from the 1000 Genomes Project (European Populations; $n=503$ ) and from Illumina Omni 5M microarrays (Krakow study population in the SiGN study; $\mathrm{n}=1,728$ ) were tested for HWE. Only variants with MAF $>0.05$ and (for the Krakow population) with $>99 \%$ successful genotypes were included in the analysis. Patterns of HWE-departure were analyzed along chromosome 17 and in selected regions with common deletions (esv2657253 on chromosome 3 and the common GSTM1/GSTM2 deletion polymorphism on chromosome 1). RESULTS: In the 1000 GP database 188918 small genetic variants on chromosome 17 were analyzed $(25,495$ indels and 163,423 SNPs). One thousand two hundred eighty (5.0\%) indels and 2,594 (1.6\%) SNPs showed significant $(p<\mathrm{E}-05)$ departure from HWE in the European population. Along selected chromosome regions, the distribution of SNPs violating HWE in the 1000 GP European sample as well as in the Krakow study sample was highly non-random and co-localized with deletion polymorphisms. On the Illumina array, regions with common structural variants showed significant reduction of SNP density. CONCLUSIONS: The increased rate of HWE-violating indels, compared to SNPs, suggests that genotype error may cause HWE-departure. However, genotyping error was not the only cause of departure from HWE, since presence of common deletions resulted also in HWE-departure. HWE-biased selection of SNPs of the Illumina array and stringent HWE filtering of observed genotypes may result in selective reduction of SNP density and failure to detect copy number variation (CNV). Exclusion of SNPs with missing genotypes may further amplify the bias against CNV detection since SNPs within common deletions have null-alleles in all homozygous carriers.

Disclosure: None.

\subsection{3}

Inbreeding associates with unfavorable outcome after ischemic stroke Eyad Hayani ${ }^{2}$; Bowang Chen ${ }^{2}$; Steve Bevan ${ }^{3}$; Christina Jern ${ }^{4}$; Jordi Jimenez-Conde ${ }^{5}$; Jin-Moo Lee $e^{6}$ Robin Lemmens ${ }^{7}$; Jonathan Rosand ${ }^{8}$; Natalia Rost ${ }^{8}$; Vincent Thijs ${ }^{9}$; Dan Woo ${ }^{10}$; Braxton Mitchell ${ }^{11}$; Steven Kittner ${ }^{12}$; John W. Cole ${ }^{12}$; Arne Lindgren $^{13}$; Caspar Grond-Ginsbach ${ }^{1}$

${ }^{1}$ Department of Neurology, University of Heidelberg, Germany; ${ }^{2}$ Department of Biology, South University of Science and Technology of China, Shenzhen, China; ${ }^{3}$ School of Life Science, University of Lincoln, UK; ${ }^{4}$ Th Sahlgrenska Academy at University of Gothenburg, Sahlgrenska University Hospital Gothenburg, Sweden; 5IMIM-Parc de Salut Mar, Universitat Autonoma de Barcelona, Spain; 'Washington University School of Medicine, St. Louis; ${ }^{7} \mathrm{KU}$ Leuven, Department of Neurology, Belgium; ${ }^{8}$ Center for Human Genetic Research, Massachusetts General Hospital, Boston; 'Department of Neurology Austin Health, and Melbourne Brain Massachusetts General Hospital, Boston; ${ }^{9}$ Department of Neurology Austin Health, and Melbourne Brain
Center, Florey Institute of Neuroscience and Mental Health, Heidelberg, Australia; ${ }^{\circ}$ Department of Neurology Center, Florey Institute of Neuroscience and Mental Health, Heidelberg, Australia; ${ }^{10}$ Department of Neurology
and Rehabilitation Medicine, University of Cincinnati, OH; ${ }^{11}$ Department of Neurology, Veterans Affairs Medical Center and Department of Medicine, University of Maryland School of Medicine, Baltimore; ${ }^{12} \mathrm{De}-$ partment of Neurology, Veterans Affairs Medical Center and Department of Neurology, University of Maryland School of Medicine, Baltimore; and ${ }^{13}$ Department of Clinical Sciences Lund, Neurology, Lund University and Department of Neurology, Skåne University Hospital, Sweden.

OBJECTIVE: To examine if inbreeding detected by CNV analysis is related to outcome after ischemic stroke. BACKGROUND: Inbreeding associates with various early and late onset conditions, including cardiovascular risk. In the current study, we hypothesized that inbreeding increases the risk for unfavorable outcome after ischemic stroke. DESIGN/ METHODS: Long runs of homozygosity ( $\mathrm{LRoH})$ were identified in Illumina Omni $5 \mathrm{M}$ microarrays from ischemic stroke patients from 7 SiGN-centers. False positive findings, due to large deletions, were excluded by visual inspection of all cases. Regions with common LRoH (pericentromeric heterochromatin, MHC locus) were excluded. Subjects with total length of autozygosity longer than $35 \mathrm{Mb}$ were classified as inbred, i.e., being offspring of related parents (second-degree cousins or more closely related). Outcome of patients with or without related parents was compared. RESULTS: The study samples comprised 2,792 microarrays from ischemic stroke patients with information on age, sex, NIHSS on admission mRS at 2-5 months and Center of recruitment. After exclusion of low quality microarrays and of patients with somatic mosaics the final study sample included 2,622 patients. Inbreeding was detected in 97 patients $(3.7 \%)$. Twenty-eight $(29 \%)$ inbred patients had unfavorable outcome (mRS 4-6), compared to $506(20 \%)$ of the remaining patients $(p=0.040)$. In a logistic regression model, unfavorable outcome was associated with parental relatedness $(p=0.008$; odds ratio $2.0 ; 95 \%$ confidence interval 1.2-3.3) and independent from age, sex, stroke severity and Center of recruitment. CONCLUSIONS: In our study sample, inbreeding was an independent predictor of unfavorable outcome after ischemic stroke. This finding suggests that recessive genetic variants contribute to outcome after ischemic stroke. However, socio-economic factors may be significant confounders of the observed association, since poverty associates with higher inbreeding rates as well as with unfavorable outcome after ischemic stroke. Disclosure: None.

\subsection{4}

Genetic imbalance is associated with poorer outcome after ischemic stroke Dorothea Pfeiffer ${ }^{2 *}$; Bowang Chen ${ }^{2 *}$; Steve Bevan ${ }^{3}$. Christina Jern ${ }^{4}$; Jordi Jimenez-Conde ${ }^{5}$; Jin-Moo Lee ${ }^{6}$; Robin Lemmens ${ }^{7}$; Jonathan Rosand ${ }^{8}$; 
Natalia Rost ${ }^{8}$ : Vincent Thij ${ }^{9}$; Dan Woo $^{10}$; Braxton Mitchell ${ }^{11}$; Steven Kittner ${ }^{12}$;ohn W. Cole ${ }^{12}$; Arne Lindgren ${ }^{13}$; Caspar Grond-Ginsbach ${ }^{1}$

*These authors contributed equally and should be considered co-first authors.

${ }^{1}$ Department of Neurology, University of Heidelberg, Germany; ${ }^{2}$ Department of Biology, South University of Science and Technology of China, Shenzhen, China; ${ }^{3}$ School of Life Science, University of Lincoln, UK; ${ }^{4}$ The Sahlgrenska Academy at University of Gothenburg, Sahlgrenska University Hospital Gothenburg, Sweden; 'IMIM-Parc de Salut Mar, Universitat Autonoma de Barcelona, Spain; 'Washington University School of Medicine, St. Louis; ${ }^{7} \mathrm{KU}$ Leuven, Department of Neurology, Belgium; ${ }^{8}$ Center for Human Genetic Research, Massachusetts General Hospital, Boston; ${ }^{9}$ Department of Neurology Austin Health, and Melbourne Brain Center, Florey Institute of Neuroscience and Mental Health, Heidelberg, Australia; ${ }^{10}$ Department of Neurology and Rehabilitation Medicine, University of Cincinnati, $\mathrm{OH} ;{ }^{11}$ Department of Neurology, Veterans Affairs Medical Center and Department of Medicine, University of Maryland School of Medicine, Baltimore; ${ }^{12} \mathrm{De}-$ partment of Neurology, Veterans Affairs Medical Center and Department of Neurology University of Maryland School of Medicine, Baltimore; and ${ }^{13}$ Department of Clinical Sciences Lund, Neurology, Lund University and Department of Neurology, Skåne University Hospital, Sweden.

OBJECTIVE: To examine if genetic imbalance regarding CNVs is related to stroke outcome. BACKGROUND: In the CADISP (Cervical artery dissection and ischemic stroke patients) sample ( $\mathrm{n}=816$; age $=44 \pm 10$ years), genetic imbalance was an independent predictor of outcome after ischemic stroke. We validated this association in an independent study sample of common stroke patients and explored the role of imbalance in specific gene groups involved in predefined biological pathways. DESIGN/METHODS: Using Illumina Omni5 microarrays of 2,622 ischemic stroke patients from 7 SiGN centers, we compared patients with favorable (mRS $0-2$ ) and unfavorable (mRS 3-6) outcome after 3 months. Genetic imbalance was defined as the total number of genes in deletion or duplication, great imbalance as imbalance of $>10$ genes. Multivariable logistic regression models were used to investigate the association between genetic imbalance and outcome and to adjust for age, sex, stroke severity (NIHSS) and center of recruitment. Predefined gene groups were selected from the GeneOntology (GO) database. RESULTS: Outcome was unfavorable in 928 of 2,622 (35.4\%) patients. Twenty-three (2.3\%) of the patients with unfavorable outcome had great imbalance, compared to $19(1.1 \%)$ of the 1694 patients with favorable outcome $(p=0.008)$. After adjustment for age, sex, stroke severity and center, great imbalance was an independent predictor of outcome $(p=0.001$; odds ratio $[\mathrm{OR}] 3.26$; $95 \%$ confidence interval [CI] 1.60-6.65). Genetic imbalance in general showed the same effect $(p=0.002$; OR 1.06; 95\% CI 1.02-1.10). Associations of unfavorable outcome with imbalance in GO gene groups associated with immune response $(p=0.002)$ and neurotransmitter secretion $(p=0.001)$ are currently being explored. CONCLUSIONS: The results validate and extend the findings in the CADISP study and confirm that genetic imbalance is an independent predictor of unfavorable outcome 3 months after stroke.

Disclosure: None.

\subsection{5}

Intracranial aneurysm disease modeling and Aneurysm

Data Repository Philippe Bijlenga, PD ${ }^{1,4}$; Sandrine Morel, PhD ${ }^{1,2,4}$; Sabine Schilling ${ }^{3}$; Sven Hirsch ${ }^{3,4}$; Daniel Rüfenacht ${ }^{4,5}$

${ }^{1}$ Neurosurgery, Geneva University Hospital; ${ }^{2}$ Pathology and Immunology, University Medical Center Faculty of Medicine of Geneva; ${ }^{3}$ Institute of Applied Simulation, Zurich University of Applied Sciences; ${ }^{4}$ SwissNeuroFoundation; and ${ }^{5}$ Interventional Neuroradiology, Klinik Hirslanden, Zürich.

OBJECTIVE: The purpose is to improve the management of patients suffering of cerebrovascular diseases and in particular intracranial aneurysms. BACKGROUND: Intracranial aneurysms affect $3 \%$ of the population. Due to advances in screening and cerebro-vascular imagining, the diagnosis is shifted from symptomatic patients to asymptomatic individuals and often is an incidental finding. When excluding the outcome due to aneurysm rupture, complications resulting in ineffective treatment, intervention associated morbidity and mortality are significant but relatively rare ( $4 \%$ and $<1 \%$, respectively). Nevertheless risks associated with intervention justify that only half the people incidentally diagnosed are offered interventions to secure the aneurysm and the others are prospectively observed with regular imaging. Selection of cases that would or not benefit treatment remains a challenge. Most centers are confronted to small yearly case loads (typically 100 to 200 cases per year). Concomitantly, clinicians are confronted to continuous developments of new decision models, treatment techniques and devices. Due to the low case loads, the evaluation of benefits associated with innovation requires multicenter and interdisciplinary collaboration. The understanding of the context associated with the management of each lesion requires the capture of multiple and heterogeneous descriptors measured by many different professionals. Because affected individual and despite treatment remain at risk all their life, the evaluation of the performance and quality of the disease management requires a quantification of health integrated over life. DESIGN/METHODS: The SNF develops a disease model on the basis of the "aneurysm life cycle" proposed by Humphrey. The model is divided in successive stages by disease markers and relevant factors are associated at each stage with probabilities to evolve to different other stages. The overall progression is modeled according to a probabilistic Swiss cheese modeling. To progressively improve the accuracy of the model a dedicated Data Repository (AneurysmDataBase) has been developed using a specific Clinical Reference Information Model (CRIM) that is regularly harmonized globally as part of the National Institute of Neurological Disorders and Stroke Common Data Elements efforts. RESULTS: The safety of a management protocol for patients diagnosed with unruptured intracranial aneurysms (IA) based on IA location and size, familial history, and risk factors as well as aneurysm growth or significant change in headaches pattern on follow-up was assessed. The yearly probability for IA patients to have an aneurysm growth is below $3 \%$. Factors associated with a higher risk are female gender, hypertension. Association of growth with location and size is similar to what was previously reported for rupture. The predictive power of a classifier based on PHASES score was assessed for the predication of aneurysm growth, decision to intervene and rupture. Area under the Receiver Operator Curve are $0.72,0.73,0.744$, respectively. We propose a modified PHASES score including aneurysm growth as seventh factor. An aneurysm classifier based an aneurysm dome shape analysis has been developed and predictive power assessed. Latest results will be presented. The incidence, severity, treatment, complications and outcome of patients suffering an aneurysm rupture was measured consecutively and prospectively at the national scale in Switzerland. The specific role of smoking on aneurysm formation, growth and rupture was studied. CONCLUSIONS: The SwissNeuroFoundation provides and infrastructure that allows the harmonization, aggregation and analysis of heterogenous data including genetics, gene expression, imaging, flow
Swiss cheese effect

Rupture trigger

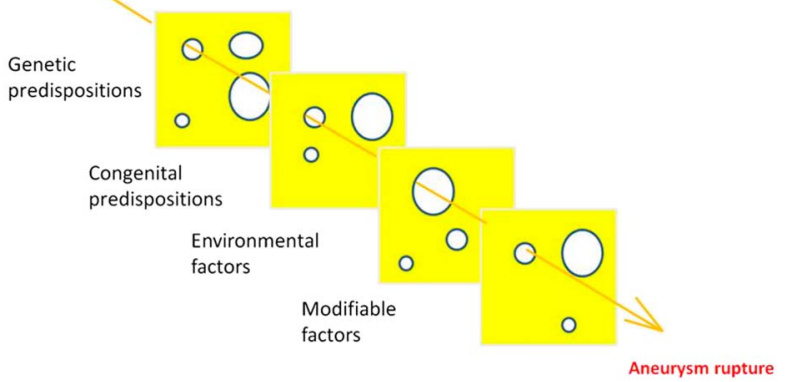

2016.045: Intracranial aneurysm disease modeling and Aneurysm Data Repository

simulations, clinical observation and long term follow-up acquired in different institutions allowing the development of a bio-mechanical and statistical model of the disease. Study Supported By: Study was performed within the framework of the AneuX project supported by SystemsX.ch, and evaluated by the Swiss National Science Foundation (2014/ 261). The study was sponsored by the Swiss NeuroFoundation. We acknowledge the contributions of the Clinical Research Center, University Hospital and Faculty of Medicine, Geneva.

Disclosure: None.

\subsection{6}

\section{Replication of two new GWAS-identified loci associated} with ischemic stroke in the Italian CEDIR Cohort Eliana Sammali, PhD ${ }^{\prime}$; Roberta Carriero, $\mathrm{PhD}^{2}$; Marina Mola Caminal, PhD $\mathrm{P}^{3}$ METASTROKE collaboration: National Institute of Neurological Disorders and Stroke Stroke Genetics Network (SiGN); GODs Study; Daniele Merico, PhD" ; Jordi Jiménez-Conde, $\mathrm{MD}^{3}$; Silvia Bione, PhD'; Maria G Franzosi, PhD ; Emilio Ciusani, $P h D^{6}$; Eugenio A. Parati, $M D^{I}$; Giorgio B. Boncoraglio, $M D^{\prime}$

${ }^{1}$ Department of Cerebrovascular Diseases, Fondazione IRCCS Istituto Neurologico Carlo Besta, Milano, Italy; ${ }^{2}$ Istituto di Genetica Molecolare, Consiglio Nazionale delle Ricerche (CNR), Pavia, Italy; ${ }^{3}$ Department of Neurology, Neurovascular Research Group, IMIM-Hospital del Mar (Institut Hospital del Mar d'Investigacions Mediques), Universitat Autonoma de Barcelona/DCEXS Universitat Pompeu Fabra, Barcelona, Spain; ${ }^{4}$ The Centre for Applied Genomics (TCAG), The Hospital for Sick Children, Toronto, ON, Canada; ${ }^{5}$ Department of Cardiovascular Research, IRCCS - Istituto di Ricerche Farmacologiche Mario Negri, Milano, Italy; and ${ }^{6}$ Laboratory of Clinical Investigations, Fondazione IRCCS Istituto Neurologico Carlo Besta, Milano, Italy.

OBJECTIVE: To replicate the association of 2 new loci for ischemic stroke found in the Italian CEDIR cohort. BACKGROUND: To date, there are no published genome-wide association study (GWAS) for ischemic stroke in Italian cases. DESIGN/METHODS: We performed a GWAS for 383 ischemic stroke Italian patients from the CEDIR cohort and 956 Italian controls from the PROCARDIS study. After quality control, we analyzed 487758 SNPs. We found a genome-wide significant association $\left(p<1 \times 10^{-7}\right)$ for 2 new SNPs, one of which (rs4780144) leads to a missense mutation in the RYR3 gene. We aimed at replicating in the Metastroke data set these findings for ischemic stroke. We also aimed at testing in the SiGN and GODs cohorts if rs 4780144 is associated with stroke severity or stroke recovery. RESULTS: We requested genotypic data for a total of 14 SNPs in the 2 selected loci, as the ones showing association $p<5 \times 10^{-3}$ and among which are the 2 with genomewide significance. As the minor allele frequencies of the 2 associated SNPs are low/ moderate, we tried to avoid genetic heterogeneity bias by testing all the associated variant in the candidate loci. For each of the 14 SNPs required, we received the summary data of the association studies used for the meta-analysis (i.e., effallele, beta, sebeta) and with the stroke subtype indicated. We transformed the beta/sebeta values into the corresponding OR with $95 \% \mathrm{CI}$ and we evaluated the ORs for each SNP in each study. For none of the 14 SNPs, we obtained evidence of replication for ischemic stroke or stroke severity. Finally, the rs 4780144 SNP is not associated with stroke recovery, but 3 SNPs in the RYR3 locus are significantly associated $\left(p<5 \times 10^{-2}\right)$ with stroke outcome at 3 months in the SiGN cohort. CONCLUSIONS: RYR3 seems a good candidate gene for ischemic stroke recovery and we will try to replicate our findings in a new Italian cohort of 450 ischemic stroke patients.

Study Supported By: For the CEDIR cohort, the Italian Ministry of Health, grant GR2011-02347041. For the PROCARDIS FP6 LSHM-CT-2007-037273.

Disclosure: None.

\subsection{7}

Genetics of early-onset ischemic stroke consortium: A call for collaboration Steven Kittner, $M D^{1}$; Christina Jern, $M D, P h D^{2}$ Giorgio B Boncoraglio, $M D^{3}$; Danish Saleheen, MBBS, PhD ${ }^{4}$; Michiaki Kubo, MD, $P h D^{5}$; Anne-Katrin Giese, $M D^{6}$; Arndt Rolfs, $M D^{7}$; Martin Dichgans, $M D^{8}$, Konstantin Strauch, PhD'; Martina Müller-Nurasyid, MD, PhD'; Hugh Markus, $M D^{10}$; Turgut Tatlisumak, $M D, P h D^{2,11}$; Daniel Strbian, $M D^{11}$; Jukka Putaala, 
$M D^{11}$; Veikko Salomaa, $M D^{11}$; Arne Lindgren, $M D, P h D^{12}$; Olle Melander, $M D, P h D^{12}$; Stephanie Debette, $M D, P h D^{13}$; Christopher Levi, MBBS ${ }^{14}$; Elizableth Holiday, PhD ${ }^{14}$; John W. Cole, MD, MS ${ }^{l}$; Patrick McArdle, PhD $D^{\prime}$; Huichun Xu, MD, PhD $D^{I}$; Braxton Mitchell, $P h D$

'University of Maryland School of Medicine; 'Sahlgrenska Academy, the University of Gothenburg, Sweden; ${ }^{3}$ Istituto Neurologico Carlo Besta, Italy; ${ }^{4}$ University of Pennsylvania; ${ }^{5}$ RIKEN Center for Integrative

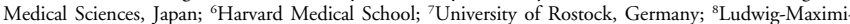
lians-Universität, Germany; ' ${ }^{\circ}$ elmholtz Zentrum München, Germany; ${ }^{10}$ University of Cambridge, UK: ${ }^{11}$ Helsinki University Central Hospital, Finland; ${ }^{12}$ Lund University, Sweden; ${ }^{13}$ University Hospital of Bordeaux, France; and ${ }^{14}$ University of Newcastle, Australia.

OBJECTIVE: The objective of this initiative is to identify both common and rare and low frequency variants associated with ischemic stroke ages 18-54 years, in order to disclose novel biological mechanisms underlying stroke risk that can be targets for preventive interventions. BACKGROUND: A strong genetic contribution to early-onset stroke has been consistently demonstrated, with early-onset ischemic stroke having both greater familial aggregation and a higher heritability than older-onset stroke. Numerous monogenic conditions presenting with the phenotype of early-onset ischemic stroke have been identified, raising the question as to whether other less penetrant variants in these genes or pathways might confer risk to early-onset stroke. As with early-onset forms of other complex diseases, the genetic contribution to stroke is likely to include some larger effect rare variants as well as smaller-effect common variants. However, GWAS studies of early-onset stroke have been few and limited by small sample sizes. Furthermore, prior studies have only considered common genetic variants, thus failing to take advantage of likely differences in the genetic architecture between older and younger stroke populations. DESIGN/METHODS: Genome-wide analyses will be conducted within each of the participating studies, and then combined across studies in a meta-analysis. For identification of rare variants, we will utilize both single variant and burden testing approaches. In addition, to these agnostic approaches, we will perform a targeted analysis of both common and rare variants in the prothrombotic pathway, based in part on results from deep venous thrombosis GWAS studies. RESULTS: To date, 15 studies with GWAS data on over 12,000 ischemic stroke cases and 65,100 controls and exome chip data on over 10,300 stroke cases and 41,300 controls have agreed to participate. All cases are ages 18-44 years of age. CONCLUSIONS: There is enthusiasm in the stroke and stroke genetics communities to collaborate on this project. Other studies with existing GWAS and/or exome chip data on cases and controls are invited to join this effort. Study Supported By: Pending.

Disclosure: None.

\subsection{8}

\section{COL4A2 variants are associated with both lacunar} ischemic stroke and deep ICH: Collaborative meta-analyses of common genetic variation in six rare familial cerebral small vessel disease genes among 21,500 stroke cases and 41,000

controls Kristiina Rannikmäe, $M D^{\prime}$; Vhinoth Sivakumaran ${ }^{2}$; Henry Millar ${ }^{2}$; Rainer Malik, PhD $D^{3}$; Tushar Dave, MS $c^{4}$; Martin Dichgans, MD, PhD ${ }^{3,5}$; Mike Chong, MSc'; Braxton D. Mitchell, MPH, PhD'; Guillaume Pare, MD, MSc, FRCPC $C^{6,7}$; Farid Radmanesh, MD, $M P H^{8-11}$; Jonathan Rosand, MD, MSt ${ }^{8-11}$; Sudha Seshadri, $M D^{12,13}$; Matthew Traylor, PhD ${ }^{14}$; Brad B. Worrall, MD, MScls; Daniel Woo, MD ${ }^{16}$; Cathie L.M. Sudlow, BMBCh, MSc, DPhil FRCPE ${ }^{1,17}$; On behalf of the Stroke Genetics Network (SiGN); METASTROKE Collaboration: The International Stroke Genetics Consortium (ISGC)

${ }^{1}$ Centre for Clinical Brain Sciences, University of Edinburgh, UK; ${ }^{2}$ College of Medicine \& Veterinary Medicine University of Edinburgh, UK: ${ }^{3}$ Institute for Stroke and Dementia Research, Klinikum der Universität München, Germany; ${ }^{4}$ Department of Medicine and Program for Personalized and Genomic Medicine, University of Maryland School of Medicine Baltimore; ${ }^{5}$ Munich Cluster for Systems Neurology (SyNergy), Germany; ${ }^{6} \mathrm{De}-$ partment of Pathology and Molecular Medicine, McMaster University, Hamilton, ON, Canada; ${ }^{7}$ Departmen of Clinical Epidemiology and Biostatistics, McMaster University, Hamilton, ON, Canada; ${ }^{8}$ Center for Human Genetic Research, Massachusetts General Hospital (MGH), Boston; ${ }^{\top}$ J. Philip Kistler Stroke Research Center, Department of Neurology, MGH, Boston, MA; ${ }^{10}$ Division of Neurocritical Care and Emergency Neurology, Department of Neurology, MGH, Boston, MA; ${ }^{1}$ Program in Medical and Population Genetics, Broad Institute, Cambridge, MA; ${ }^{12}$ Boston University Schools of Medicine and Public Health, Boston, MA; ${ }^{13}$ Framingstitute, Cambridge, MA; ${ }^{12}$ Boston University Schools of Medicine and Public Health, Boston, MA; ${ }^{13}$ Framing-
ham Heart Study, Framingham, MA; ${ }^{14}$ Department of Clinical Neurosciences, University of Cambridge, UK ham Heart Study, Framingham, MA; ${ }^{14}$ Department of Clinical Neurosciences, University of Cambridge, UK,
${ }^{15}$ Departments of Neurology and Public Health Sciences, University of Virginia, Charlottesville; ${ }^{16}$ Department of Neurology, University of Cincinnati College of Medicine, OH; and ${ }^{17}$ Institute for Genetics and Molecula Medicine, University of Edinburgh, UK.

OBJECTIVE: We aimed to determine whether common genetic variants in familial cerebral SVD genes confer risk of sporadic cerebral SVD. BACKGROUND: Mutations in several genes cause rare familial forms of cerebral small vessel disease (SVD), but the genetic determinants of sporadic cerebral SVD, thought to underlie most lacunar ischemic strokes (IS) and deep intracerebral hemorrhages (ICH), remain largely unknown. DESIGN/METHODS: We conducted meta-analyses of genotype data among individuals of European ancestry to determine associations of common single nucleotide polymorphisms (SNPs) in 6 familial cerebral SVD genes (COL4A1, COL4A2, NOTCH3, HTRA1, TREX1, and CECR1) with: ICH (deep, lobar, all; 1,878 cases, 2,830 controls) and IS (lacunar, cardioembolic, large vessel disease, all; 19,569 cases, 38,159 controls). We applied a range of data quality filters, and set statistical significance thresholds that accounted for multiple testing and linkage disequilibrium between SNPs. RESULTS: A locus in COL4A2 was significantly $(p<3.5$ $\times 10^{-4}$ ) associated with both lacunar IS (lead SNP rs9515201: OR 1.17; $95 \% \mathrm{CI}$ $\left.1.11-1.24, p=6.62 \times 10^{-8}\right)$ and deep ICH (lead SNP rs4771674: OR 1.28; $95 \%$ CI $\left.1.13-1.44 ; p=5.76 \times 10^{-5}\right)$. A SNP in HTRA1 was significantly $\left(p<5.5 \times 10^{-4}\right)$ associated with lacunar IS (rs79043147: OR 1.23, 95\% CI $1.10-1.37, p=1.90 \times$ $10^{-4}$ ), and less robustly with deep ICH. There was no clear evidence for association of common variants in: either COL4A2 or HTRA1 with non-SVD strokes; or in any of the other genes with any stroke phenotype (figure). CONCLUSIONS: These result provide evidence of shared genetic determinants of distinct ischemic and hemorrhagic cerebral SVD stroke phenotypes, and offer new insights into the causal mechanisms of cerebral SVD.

Disclosure: None.

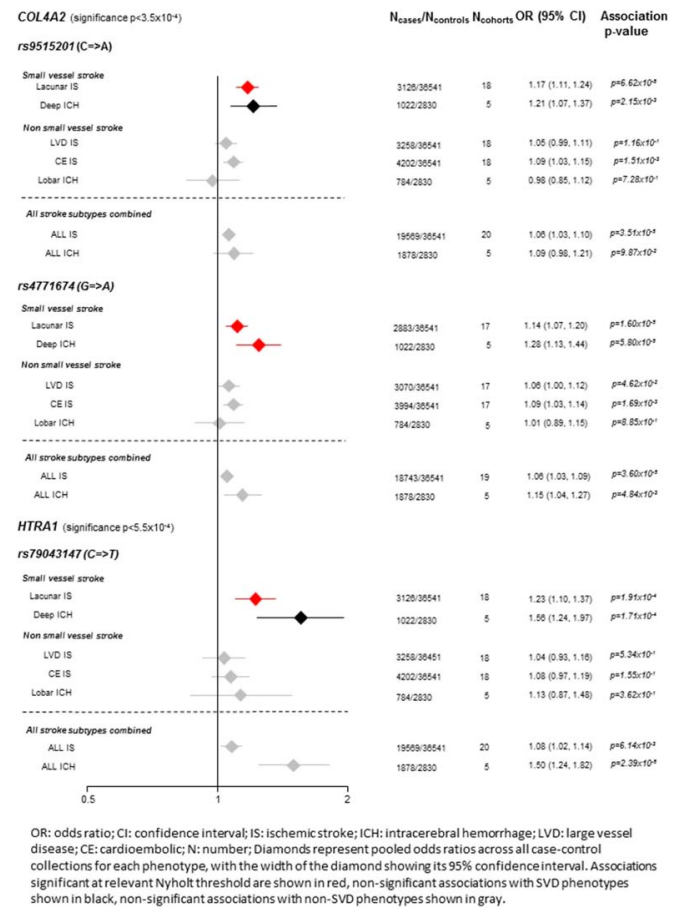

2016.048: COL4A2 variants are associated with both lacunar ischemic stroke and deep ICH: Collaborative meta-analyses of common genetic variation in six rare familial cerebral small vessel disease genes among 21,500 stroke cases and 41,000 controls

\subsection{9}

Genetics of stroke in etiology and prognosis after stroke in young adults Frank-Erik de Leeww, MD, PhD'; Anil M. Tuladhar, $M D^{I}$; Ewoud J. van Dijk, $M D, P h D^{t}$; Hennie Schoonderwaldt, MD, PhD ; Loes C.A. Rutten-Jacobs, PhD ${ }^{2}$; Catharina J.M. Klijn, $M D, P h D^{1}$

${ }^{1}$ Radboud University Medical Centre, Donders Institute for Brain, Cognition and Behaviour, Centre for Cognitive Neuroscience, Department of Neurology, Nijmegen, the Netherlands; and ${ }^{2}$ University of Cambridge, Department of Clinical Neurosciences, Neurology Unit, Cambridge, UK.

OBJECTIVE: To contribute to the development of consortia that specifically aim to investigate the genetics in its broadest sense of "young stroke" etiology and prognosis. BACKGROUND: There is a certain genetic contribution to stroke etiology and prognosis, that is presumably even larger in "young stroke." To answer these questions large consortia of extremely well phenotyped and radiology assessed patients, with ideally a long-term follow up need to be established. DESIGN/METHODS: Future study; a prospective cohort study of patients with a TIA, ischemic stroke or intracerebral hemorrhage aged between 18 and 50 years of age. Single center study with baseline data collection starting from 1980 to 2010; mean follow up 11 years. Apart from detailed baseline phenotyping (risk factors, etiology) at follow-up neuroimaging was performed and DNA was collected of approximately 500 patients. Next there is the ODYSSEY Study, a multicenter prospective cohort study in the Netherlands that investigates causes and consequences of young stroke (imaging proven TIA, ischemic and hemorrhagic stroke). We will collect 1,500 cases, currently about 620 patients included. Detailed phenotyping is done at baseline (starting 2013) and at least 3 years of follow up will be obtained. DNA is available and there is medical ethics approval for participation in international genetic consortia. RESULTS: Inclusion of the latter ODYSSEY Study is ongoing and we expect to finish inclusion in 2020, follow up will be completed in 2023 . CONCLUSIONS: We expect that these thousands of extremely well phenotyped patients will understand our knowledge on the genetic contribution to the etiology and prognosis after stroke in young adults.

Study Supported By: Declaration of interests Prof. de Leeuw is supported by a clinical established investigator grant of the Dutch Heart Foundation (grant number 2014 T060) and by a VIDI innovational grant from The Netherlands Organisation for Health Research and Development (ZonMw grant 016-126-351). Dr. van Dijk received a personal fellowship from the Dutch Brain Foundation (H04-12; F2009(1)-16). Prof. Klijn is supported by a clinical established investigator grant of the Dutch Heart Foundation (grant number 2012 T077), and an Aspasia grant from The Netherlands Organisation for Health Research and Development (ZonMw grant 015-008-048).

Disclosure: None.

\subsection{0}

Whole genome sequence analysis of brain MRI measures in the Framingham Study Chloé Sarnowski, $P h D^{\prime}$; Claudia L. Satizabal, PhD ${ }^{2,3}$; Charles DeCarli, MD'; Achilleas N. Pitsillides, PhD'; Alexa Beiser, PhD ${ }^{1,2,3}$; Anita L. DeStefano, PhD ${ }^{1,2,3}$; Josée Dupuis, PhD ${ }^{1,2}$; Sudha Seshadri, MD ${ }^{2,3}$ 
${ }^{1}$ Department of Biostatistics, Boston University School of Public Health, MA; ${ }^{2}$ The National Heart, Lung, and Blood Institute's Framingham Heart Study, Framingham, MA; ${ }^{3}$ Department of Neurology, Boston University School of Medicine, MA; and ${ }^{4}$ Department of Neurology, University of California at Davis. OBJECTIVE: Genome-Wide Association Studies have identified common genetic variant with modest effect sizes, and most associated variants reside in non-coding regions. We sought to identify rare variants influencing brain MRI measures in the Framingham Study (FHS) by performing Whole Genome Sequence (WGS) analyses within the Trans-Omics for Precision Medicine (TOPMed) Program. BACKGROUND: Brain MRI measures such as Total Cerebral Brain Volume (TCBV), Hippocampal Volume (HPV) and White Matte Hyperintensities (WMH) are endophenotypes of Alzheimer disease (AD) and vascular injury. Identifying loci influencing these measures may reveal new genes and biological mechanisms underlying these diseases. DESIGN/METHODS: A total of 2,180, 2,170, and 1,667 individuals were included in the WGS analyses of TCBV, HPV and WMH respectively. Rank-normalized residuals from mixed effect linear regression models adjusted for sex, age, and total intracranial volume were tested for association with individual variants while accounting for familial relatedness. Gene-based tests were conducted for rare variants (Mino Allele Frequency (MAF) lower or equal to $1 \%$ ) with SKAT or burden test within genes using (1) a sliding-window approach, (2) a selection of functional exonic variants, or (3) all variants. RESULTS: WGS analyses revealed new loci at the genome-wide level $\left(p \leq 5 \times 10^{-8}\right)$ in $1 \mathrm{p} 21$ for TCBV between RWDD3 and PTBP2 (MAF $\left.=0.005, p=10^{-8}\right)$ and in $16 \mathrm{q} 23$ for HPV within LOC102724084 (MAF $\left.\sim 5 \%, p=2.7 \times 10^{-8}\right)$. The known associations in $12 \mathrm{q} 24$ for HPV ( $p$ lower or equal to 0.01 ) and in $17 \mathrm{q} 25$ for WMH $\left(p \leq 6.7 \times 10^{-6}\right)$ were observed. Gene-based tests applied to genes and performed using a sliding-window approach detected genome-wide associations $\left(p \leq 2.3 \times 10^{-6}\right)$ in new loci with SKAT for TCBV (FCHO2 in 5q13, UNC5D in 8p12, CCDC33 in 15q24) and HPV (LRRTM4 in 2p12) and with the burden test for TCBV (C9orf84 in 9q31, UBL3 in 13q12-q13, SYT3 in 19q13) and WMH (ALCAM in 3q13, GBA3 in 4p15). SKAT method applied to all variants within genes detected SOCS7 in $17 \mathrm{q} 12$ significantly associated with TCBV. CONCLUSIONS: In conclusion, WGS-wide search for brain MRI measures reveals intriguing new loci influencing brain volumes.

Study Supported By: R01 AG033193, R01 AG008122, R01 AG049505, R01 AG049607, R01 NS017950, R01 AG054076, FHS contract HHSN268201500001I.

Disclosure: None.

\subsection{1}

The 1-1 haptoglobin phenotype: Perihematoma edema progression in acute intracranial hemorrhage Michael $R$ Halstead; William Andrew Mould; Kevin N. Sheth; Jonathon Rosand; Richard Thompson; Andrew Levy; Daniel F. Hanley; Joshua N. Goldstein; Paul Nyquist

Johns Hopkins Hospital, Baltimore Yale School of Medicine and Yale New Haven Hospital, CT; Massachusetts General Hospital, Boston; Technion Israel Institute of Technology, Hafia; and Johns Hopkins Bloomberg School of Public Health.

OBJECTIVE: To determine if the Haptoglobin 1-1 phenotype has a different rate of PHE compared to 2-1 and 2-2 and to see if the different rates of PHE related to Haptoglobin phenotype affect clinical outcomes. BACKGROUND: Haptoglobin phenotype affects in flammation and may change rate of perihematoma edema formation (PHE) affecting outcomes. We reported better functional recovery in patients with intracranial hemorrhage (ICH) and the Haptoglobin 1-1 phenotype. This difference may be mediated through Haptoglobin 1-1 effects on PHE. DESIGN/METHODS: We determined Haptoglobin phenotype, ICH volume, and $\mathrm{PHE}$ in individuals identified prospectively from $\mathrm{ICH}$ cohorts, at Johns Hopkins University Hospital (JHU) and the Massachusetts General Hospita (MGH) using established CT methodology. PHE were measured as differences in CT ICH edema volume divided by hours between scans. Associations of Haptoglobin phenotype (1-1 compared to 2-1, 2-2) and rate of PHE (cc/h) using multivariate analysis adjusting for age, sex, race, diabetes (DM), hypertension (HTN), LDL cholesterol $>130 \mathrm{~mm} / \mathrm{di}$ (HDL), smoking, prior $\mathrm{ICH}$, and use of antiplatelet or systemic anticoagulation were determined. RESULTS: Eighty-seven patients were included with the following characteristics: Mean age 61.6 (15), 44 (50\%) females, 28 (32\%) AA, 64 (74\%) HTN, 17 (20\%) HDL, 22 (25\%) active smokers, $29(33 \%)$ on some form of anticoagulation/anti platelet and $9(10 \%)$ with prior $\mathrm{ICH}$. We included $57 \mathrm{JHU}$ and $30 \mathrm{MGH}$ participants with different baseline characteristics. There were 9 subjects with Haptoglobin genotype 1-1, 45 with 2-1 and 33 with 2-2, Mean PHE varied within each group. Fully adjusted estimated mean PHE for 1-1 haplotype was $1.84 \mathrm{cc} / \mathrm{h}(95 \% \mathrm{CI} 0.87-2.28 \mathrm{cc} / \mathrm{h})$, which was significantly greater than Haplotype 2-1 at $0.29 \mathrm{cc} / \mathrm{h}(-0.15$ to $0.72 \mathrm{cc} / \mathrm{h})$ and Haplotype $2-2$, at $0.44 \mathrm{cc} / \mathrm{h}(-0.07$ to $0.96 \mathrm{cc} / \mathrm{h})$ CONCLUSIONS: In adjusted models, Haptoglobin 1-1 phenotype on average had a significantly increased rate of PHE in the early stages of ICH onset, opposite that predicted by our biological model. Our study suggests Haptoglobin haplotype is associated with PHE and may account for variability in disability and mortality in ICH. Our study is limited by insufficient power to determine haplotype effect on disability and mortality and larger prospective studies are warranted.

Study Supported By: None.

Disclosure: This work submitted for presentation at the AHA International Stroke Conference in February 2017.

\subsection{2}

The genetics of stroke recovery: Is it time to revisit candidate genes? Jane M. Maguire ${ }^{1,2}$; On behalf of GISCOME Investigators

${ }^{1}$ Faculty of Health and Medicine, University of Newcastle, Callaghan, Australia; ${ }^{2}$ Hunter Medical Research Institute, Newcastle, Lambton Heights, Australia.

OBJECTIVE: To identify, appraise and describe the candidate gene evidence for the role of genetics in stroke recovery. This critical review will identify the most robust and promising candidate single nucleotide polymorphisms for stroke recovery that may be investigated in future GWAs. To describe the future directions for stroke recovery genetic research BACKGROUND: It remains difficult to predict clinical improvement or deterioration in function, psychosocial outcomes or mortality following a stroke. Current rehabilitation and recovery management approaches are advancing at a rapid rate but standard definitions and consensus around the most relevant time points to deliver therapies and best measures of outcome are hampering efforts to move this field forward (Bernhardt et al., 2016). Biomarkers that include genetic variants are thought to have potential to provide more information about recovery mechanisms and hence prognosis (Burke and Cramer, 2013; Lindgren and Maguire, 2016). Thus there is urgent need to better understand the biology that occurs post stroke and at several key time points along the recovery trajectory. This understanding would be enhanced with the addition of knowledge derived from more powerful genome wide genetic association studies. DESIGN/METHODS: A critical review of scholarly articles that measured functional ability, psychosocial outcomes and mortality in stroke patients with a case control genetic association design was conducted in April 2016. Electronic databases including MEDLINE and PubMed via National Center for Biotechnology Information website ncbi.nlm.nih.gov/ pmc. Quality appraisal was conducted and used the JBI Checklist for Case-control studies. RESULTS: The search resulted in 35 original research papers. Nine papers were excluded on abstract as these were not considered relevant and did not focus on single nucleotide associations (snp) with stroke outcomes. Only one study investigated psychosocial attributes post stroke. Carriers of the SERT gene 5-HTTLRR and STin2 were associated with a higher risk of post stroke depression. APOE rs7412 and rs429358 were associated with improved outcomes in 2 studies, whilst results for BDNF rs6265 were conflicting for both poor and favorable outcome. Data suggests that BDNF played a more significant role in motor cortex excitability. Most studies had limited samples sizes and as a consequence were underpowered. CONCLUSIONS: It is timely to revisit candidate SNPs associated with stroke outcome and with a functional role and couple this with replication in more powerful GWAs studies.

Disclosure: None.

\subsection{3}

Age-of-onset informed genome-wide association study in stroke Sara L. Pulit; On behalf of the SiGN Phenotype Committee

University Medical Center Utrecht, Department of Neurology, Brain Center Rudolf Magnus, the Netherlands.

OBJECTIVE: To identify additional stroke loci by using an age-of-onset informed approach, as developed by Matthew Traylor and others. BACKGROUND: Ischemic stroke is one of the leading causes of death worldwide, and approximately 15 million strokes occur each year. Genome-wide association studies (GWAS) have helped unravel the underpinnings of ischemic stroke; a handful of loci, including /PITX2/, /ZFHX3/, /TSPAN2/, 12q24.12, and /HDAC9/ have been revealed as robustly associated to disease. In addition, a number of clinical risk factors for stroke, including hypertension and type 2 diabetes, have also been identified and explain a fraction of the phenotypic variation observed in individuals affected by stroke. An additional key risk factor is age, as most strokes occur after age 65. Leveraging these clinical risk factors in genetic studies can potentially help further elucidate the genetic underpinnings of disease. DESIGN/METHODS: With the aim of identifying additional stroke risk loci, we have applied an age-at-onset informed approach (developed by Matthew Traylor and others) to the Stroke Genetics Network (SiGN) data set. The method generate a new phenotype for each case and control: a posterior liability score generated after conditioning on age at onset as well as disease status. Using the posterior liability phenotype, we performed genome-wide association testing in the European- and African-ancestry samples available in SiGN (up to $\sim 13,000$ cases and $\sim 30,000$ controls). We performed testing in al stroke as well as the 3 primary subtypes - cardioemobolic stroke, large artery atherosclerosis, and small artery occlusion-as determined by the CCS Causative, CCS Phenotypic, and TOAST subtyping methods. RESULTS: Analyses are ongoing, and we have identified a small handful of novel loci exceeding genome-wide significance in discovery. These loci will need to be further examined and followed up in independent samples. CONCLUSIONS: Alternate analytic approaches, such as analyzing phenotypes of posterior liabilities (which take into account clinical information) can be useful in uncovering additional loci that increase risk of stroke, and therefore help elucidate potential mechanisms of disease. Study Supported By: NIH/National Institute of Neurological Disorders and Stroke U01 NS069208.

Disclosure: None

\subsection{4}

\section{Genome-wide association studies from the Vitamin Intervention for Stroke Prevention (VISP) trial detect novel loci for recurrent stroke Keith L. Keene, $P h D^{\prime}$; Wei-Min Chen} $\mathrm{PhD}^{2}$; Fang-Chi Hsu, PhD ; Stephen R. Williams, $\mathrm{PhD}^{2}$; Michele M. Sale, PhD ${ }^{2}$; Bradford B. Worrall, $M D^{2}$; On behalf of the GARNET Collaborative Research Group

${ }^{1}$ East Carolina University, Greenville, NC; and ${ }^{2}$ University of Virginia, School of Medicine, Charlottesville; and ${ }^{3}$ Wake Forest University School of Medicine, Winston Salem, NC.

OBJECTIVE: Identify genetic determinants of recurrent stroke. BACKGROUND: Recurrent strokes, which account for $\sim 25 \%$ of all strokes annually, are more deadly and most likely to cause disability when compared to a first stroke. Unfortunately, genetic studies of recurrent stroke have been severely lacking. Our group has completed a genome-wide association study, identifying 2 novel loci associated with recurrent stroke in the Vitamin Intervention for Stroke Prevention (VISP) clinical trial population. DESIGN/METHODS: A subset of 2,100 VISP participants were genotyped on the Illumina HumanOmni1-Quad_v1-0_B BeadChip. Survival analyses, in which survival of stroke (days of follow-up to recurrent stroke) was regressed on gender, age, treatment group, and principal components 1-4, were performed. RESULTS: These analyses detected 2 genome-wide significant associations (rs6664786; $\beta=-0.62, p=1.73 \times$ $10^{-8}$ and rs $\left.2184006 ; \beta=-0.62, p=3.43 \times 10^{-8}\right)$ located in a gene sparse region of chromosome 1. Subsequent treatment-stratified analyses of recurrent stroke, using Cox proportional hazards regression survival analysis, identified a cluster of nearly a dozen SNPs on chromosome 2 with genome-wide significant associations for recurrent stroke in the low dose treatment arm (adjusting for age, sex, and the top 5 principal components). The top SNP, rs6542775 $\left(\beta=-1.966, p=2.19 \times 10^{-11}\right)$ is located downstream of the LIM and senescent cell antigen-like domains 1 (/LIMS1/) gene. 
Kaplan-Meier survival analyses by genotype for the top SNP, rs6542775 revealed that individuals in the low dose treatment arm carrying the GG genotype had significantly higher probability of longer survival (not suffering a recurrent stroke) than those with either the GA or AA genotypes $\left(p=6.1 \times 10^{-8}\right)$. In comparison, there was no statistically significant difference in the high dose arm by genotype at this SNP. CONCLUSIONS: These findings suggest that variants across these 2 regions may have implications with regards to recurrent stroke risk and potential therapeutic response.

Study Supported By: R01 NS34447; U01 HG0044381; U01 HG005157; U01HG005160.

Disclosure: None.

\subsection{5}

\section{A network approach to identification of new candidates} for stroke and stroke subtypes Stephen $R$. Williams, $P h D^{\prime}$; Qiong Yang, PhD'; Tushar Dave, $M S^{3}$; Braxton Mitchell, $P h D^{4}$; Sudha Seshadri, $M D^{5}$; Michele M. Sale, PhD $D^{6}$; Bradford B. Worrall, $M D, M S c^{\dagger}$

'Department of Neurology, University of Virginia, Charlottesville; ${ }^{2}$ Department of Biostatistics, Boston University, MA; ${ }^{3}$ School of Medicine, University of Maryland, Baltimore; ${ }^{4}$ Boston University, Department of Neurology, MA; and 'Department of Public Health, University of Virginia, Charlottesville.

OBJECTIVE: The objective of this study was to explored using network analysis to inform GWAS of stroke and stroke subtypes to identify biologically tractable candidates. BACKGROUND: Identification of the genetic contributors to stroke, stroke subtypes, and stroke risk factors is difficult because of the complex ideology of the disease(s) and investigation into more refined phenotypes such as atherosclerosis may be useful. Advanced computational techniques that can assess the impact of many genes together rather than the traditional "one gene one phenotype" approach are lacking in the field of cerebrovascular disease but may be useful to identify new biologically relevant candidates. DESIGN/METHODS: We performed a Weighted Gene Co-expression Network Analysis (WGCNA) of carotid atherosclerotic plaques paired with healthy distant tissue from 32 individuals $(63$ samples total, one removed during QC) (Data set: GSE43292). After soft thresholding, 16 distant modules of co-expressed genes were detected. Six of these modules were significantly associated with carotid atherosclerosis with 2 being distinct candidate modules. These modules are enriched for genes known to be involved in the pathogenesis of atherosclerosis and stroke; however, they also contain candidates that are novel and biologically relevant. RESULTS: Using highly connected "hub" genes, SNPs known to be eQTLs for these transcripts, as per The Genotype-Tissue Expression (GTEx) project, were used to inform GWAS of ischemic stroke and subtypes in METASTROKE and SiGN. Further, we refined our SNP list to only those known eQTLs in aorta, coronary artery, and tibial artery. This enriched our data set for SNPs known to be functionally relevant in tissues associated with stroke and atherosclerosis. Significant SNPs at the /ADAMTSL3/ locus were identified for ischemic stroke (rs2585064, $p=5.31 \times 10^{-5}$; / priori/ significance threshold $p=6.25 \times 10^{-5}$ ) as well as LVD, SVD, and CE subtypes in METASTROKE. These findings were replicated in SiGN for ischemic stroke $(p=0.017)$, and $\operatorname{LVD}(p=0.003)$, but not CE $(p=0.31)$. This gene has never been directly implicated in cerebrovascular disease but is an excellent candidate. Protein family members are known be negative regulators of von Willebrand Factor, an established risk factor for stroke. Adding to a possible pathophysiologic mechanism, /ADAMTSL3/ mRNA is reduced in carotid atheroma compared to control tissue $\left(p=5.47 \times 10^{-6}\right)$ which could lead to increased risk for clotting and thus ischemia. CONCLUSIONS: Using a novel approach in cerebrovascular genetics, these findings set the stage for expanded replication and molecular follow-up which could lead to a new target for treatment of atherosclerosis and stroke prevention.

Disclosure: None.

\subsection{6}

\section{An Epigenome Wide Association study reveals an altered methylation pattern associated with acute neurological outcome after ischemic stroke Natalia Cullell,} $M S c^{l}$; Marina Mola-Caminal, MSc'; Carolina Soriano-Tárraga, PhD'; Cristina Gallego-Fabrega, $P h D^{3}$; Elena Muiño, $M D^{\prime}$; Jerzy Krupinski, $M D$, PhD $D^{2,4}$; Nuria Torres, $M c^{l}$; Laura Heitsch, $M D^{5}$; Laura Ibañez, PhD ${ }^{6}$; Carlos Cruchaga, PhD $D^{6,7}$; Joan Montaner, $M D$, PhD ${ }^{8,9}$; Jin-Moo Lee, MD, PhD ${ }^{10}$; Jaume Roquer, $M D$, PhD ${ }^{2}$; Jordi Jimenez-Conde, $M D$, PhD $D^{2}$; Israel Fernandez-Cadenas, $P h D^{I}$

${ }^{1}$ Stroke Pharmacogenomics and Genetics, Fundació Docència i Recerca MútuaTerrassa, Hospital Mútua de Terrassa; ${ }^{2}$ Department of Neurology, Hospital del Mar; Neurovascular Research Group, IMIM (Institut Hospital del Mar d'Investigacions Mèdiques); Universitat Autònoma de Barcelona/DCEXS-Universitat Pompeu Fabra, Barcelona, Spain; ${ }^{3}$ Center for Human Genetic Research, Massachusetts General Hospital, Harvard Medical School, Boston; ${ }^{4}$ Neurology Service. Hospital Mútua de Terrassa; ${ }^{5}$ Department of Emergency Medicine, Washington University, St. Louis, MO; ${ }^{6}$ Department of Psychiatry, School of Medicine, Washington University, St. Louis, MO; ${ }^{7}$ Hope Center Program on Protein Aggregation and Neurodegeneration, Washington University, St. Louis, MO; ${ }^{8}$ Department of Neurology, Hospital Universitari Vall d'Hebron Washington University, St. Louis, MO; ${ }^{8}$ Department of Neurology, Hospital Universitari Vall d'Hebron
Barcelona, Spain; ' ${ }^{9}$ Neurology Unit Virgen del Rocio Hospital; and ${ }^{10}$ Stroke Center, Department of Neurology and the Hope Center for Neurological Disorders, Washington University School of Medicine, St. Louis, MO OBJECTIVE: Our aim was to study whether epigenetics could be associated with acute neurologic outcome in stroke patients. BACKGROUND: Acute neurologic outcome after stroke differs among patients. Different factors are involved in the prognosis of stroke patients such as age, infarct size, stroke subtype or genetics background. DESIGN/METHODS: We performed an epigenetic study in $450,000 \mathrm{CpG}$ methylation sites, using the InfiniumHuman-Methylation 450 Beadchip (Ilumina) in 727 ischemic stroke patients. We calculated the $\triangle$ NIHSS-discharge (baseline NIHSS - NIHSS at discharge) for all patients and the $\triangle$ NIHSS-24h (baseline NIHSS - NIHSS at 24 hours) was available for 118 patients. All processing, correction, normalization and analysis steps were implemented using R. After quality controls, the levels of methylation of 661 patients for $\triangle$ NIHSS discharge and 112 patients for $\triangle$ NIHSS-24 were analyzed with generalized linear model using as covariates sex, age, smoking status, batch and PCA. RESULTS: The $\triangle$ NIHSS discharge analysis identified an epigenome-wide association of a CpG site $\left(p=8 \times 10^{-8}\right)$ with neurologic outcome. This $\mathrm{CpG}$ site located on chromosome 12 regulates the expression of proteins involved in the TFG-beta signaling pathway. Additional CpG sites $(n=15)$ were identified with a nominal epigenome-wide significance $\left(p<10^{-6}\right)$. One of them was located in a gene previously related with neurologic outcome in animal models of ischemia. Another CpG site $\left(p=8 \times 10^{-6}\right)$ was found inMACROD2 gene, associated previously with covert MRI-defined brain infarcts. The $\triangle$ NIHSS-24 analysis identified a CpG site on chromosome $10\left(p=1.8 \times 10^{-7}\right)$ associated with neurologic outcome at 24 hours after ischemic stroke, the gene has been involved in cell proliferation. CONCLUSIONS: Our study found differential methylation status associated with neurologic outcome after ischemic stroke, replication analysis will be necessary to confirm those associations.

Study Supported By: Genisis NIH project and Pharmastroke project, Carlos III Institute. Disclosure: None.

\subsection{7}

Integrative analysis of hemostatic gene regulation in the human liver using a targeted sequencing

\section{approach Martina Olsson, MSc ${ }^{\prime}$; Tara M. Stanne, $\mathrm{PhD}^{\prime}$; Marcela Davila, $\mathrm{PhD}^{2}$;} Christina Jern, $M D, P h D^{l}$

${ }^{1}$ Institute of Biomedicine, Sahlgrenska Academy at University of Gothenburg, Sweden; and ${ }^{2}$ Bioinformatics Core Facility, Sahlgrenska Academy at University of Gothenburg, Sweden.

OBJECTIVE: The aim of the present study is to identify novel cis-regulatory variants and DNA methylation patterns involved in regulating hemostatic genes in the human liver. BACKGROUND: Most factors involved in hemostasis are produced in the liver. Tight regulation of these genes is crucial to avoid thrombus formation which can lead to ischemic stroke. Genetic variants together with epigenetic modifications modulate gene expression and can alter an individual's phenotype. Here, we utilize high resolution gDNA-, mRNA-, and DNA methylation sequencing (-seq) of 77 hemostatic genes in human liver tissue to investigate associations between SNPs and gene expression (expression quantitative trait loci; eQTL), SNPs and DNA methylation (methylation quantitative trait loci; meQTL) and gene expression and DNA methylation (expression quantitative trait methylation; eQTM). Candidate variants identified in this project will then be analyzed for association to ischemic stroke and other thrombotic events. DESIGN/METHODS: We have collected biopsies of healthy human liver tissue from adults undergoing liver surgery at the Sahlgrenska University Hospital $(\mathrm{n}=70)$. For the first set of samples $(\mathrm{n}=19)$ we isolated genomic DNA (gDNA) and total RNA. We designed a targeted approach in order to enrich and sequence 77 selected genes that are important for hemostasis, including upstream (5,000 bp) and downstream (500 bp) regions, and their corresponding mRNA transcripts. For RNA-seq, all potential transcript isoforms were included, as well as one negative control and 4 liver cell specific markers. Genetic variants were extracted from gDNA-seq data, expression levels were attained from mRNA-seq data, and high resolution CpG methylation status was extracted from methylation-seq data. The R package Matrix eQTL was used to assay eQTLs, meQTLs and eQTMs. RESULTS: Initial results from the quantitative trait analyses revealed approximately 17,000 cis-meQTLs (in 62 unique genes), 300 ciseQTLs (21 genes), and 300 eQTMs (19 genes). False discovery rate (FDR) was equal or less than 0.05 for all reported associations. CONCLUSIONS: In conclusion, the present study provides novel insights into the mechanistic relationships between genetic variation, DNA methylation and gene expression of hemostatic genes in the human liver. Study Supported By: The Swedish Research Council, the Swedish Heart and Lund Foundation, the Rune och Ulla Amlöv Foundation, the P-O Ahl foundation and Science for Life Laboratory's long-term bioinformatics support (WABI).

Disclosure: None.

\subsection{8}

\section{Identification of putative pathogenic CNVs in} a large cohort of patients with intracerebral hemorrhage Amanda Donatti ${ }^{I}$; Rodrigo Secolin ${ }^{l}$; Fábio R. Torres ${ }^{I}$; Leslie E. Ferreira $^{2}$; Miriam Maccarone ${ }^{3}$; Paulo H.C. França $a^{2}$ Vivian Nagel; Michelangelo Mancuso ${ }^{3}$; Norberto L. Cabral'; Benilton S. Carvalho ${ }^{4}$; Daniel Woo ${ }^{5}$ Iscia Lopes-Cendes ${ }^{I}$

${ }^{1}$ Department of Medical Genetics, School of Medical Sciences, University of Campinas-UNICAMP; and the Brazilian Institute of Neuroscience and Neurotechnology (BRAINN), Campinas, SP, Brazil; ${ }^{2}$ Department of Medicine, Regional University of Joinville-UNIVILLE, Joinville, SC, Brazil; ${ }^{3}$ Department of Experimental and Clinical Medicine, Neurological Institute, University of Pisa, Italy; ${ }^{4}$ Department of Statistics, Institute of Mathematics, Statistics and Scientific Computing, University of Campinas-UNICAMP, SP, Brazil; and ${ }^{5}$ Department of Neurology and Rehabilitation Medicine, College of Medicine, University of Cincinnati, $\mathrm{OH}$

OBJECTIVE: The aim of this study is to identify putative pathogenic CNVs in patients with ICH. BACKGROUND: Intracerebral hemorrhage (ICH), although less frequent than ischemic stroke, has a higher mortality rate. Several studies have demonstrated evidence of genetic factors influencing ICH. Copy number variations (CNVs) are common structural mutations, which have been identified as causing changes in gene expression, thus potentially leading to disease. DESIGN/METHODS: We performed microarray-based genotyping of SNPs (Genome-Wide Human SNP Array 6.0; Affymetrix Inc.) to identify CNVspresent in patients with ICH. The cohort is composed of individuals with a minimum age of 50 , divided into 2 groups: 1,053 patients with ICH and 781 healthy individuals. Patients were ascertained in 4 centers: University of Joinville, Brazil; University of Pisa, Italy; Massachusetts General Hospital, USA and University of Cincinnati, USA. CNV calls were performed by 2 algorithms, Affymetrix APT Copy Number tool and PennCNV; only CNVs identified in both were recorded. In addition, we only considered CNVs containing more than 25 markers, in order to avoid short variations that could interfere in our analysis. We determined CNV frequency by PLINK, and in an initial analysis, considered only CNVs present exclusively in patients. These were subsequently analyzed by GProfiler and METACORETM software in order to identify the main pathways related to the phenotype. RESULTS: Overall, we identified 866 $\mathrm{CNV}$ s among cases. Individual $\mathrm{CNV}$ s identified were present at a low frequency, with a maximum of 12 patients with the same CNV. GProfiler and METACORETM software identified 4 potential genes related to Ras, MAPK and Ephrin signaling pathways ( $p=1.57 \mathrm{e}-2$; 
$p=5.026 \mathrm{e}-8)$ within these CNVs: CDC42, EFNA1, EFNA3 and SHC1. CONCLUSIONS Copy number variants may identify additional genes of interest to ICH risk. Study Supported By: Brazilian Institute of Neuroscience and Neurotechnology (BRAINN) and FAPESP.

Disclosure: None.

\subsection{9}

Development of a knowledge base to support the automatic classification of a computable ischemic stroke phenotype from electronic medical

records Danielle L. Mowery, PhD, MS ${ }^{1}$; Brent Hill, PhD'; Wendy W. Chapman $\mathrm{PhD}^{\prime}$; Lisa Cannon-Albright, PhD, MStat ${ }^{3}$; Jennifer J. Majersik, $M D, M S^{4}$

${ }^{1}$ Department of Biomedical Informatics; ${ }^{2}$ Clinical Neurosciences Center; ${ }^{3}$ Division of Genetic Epidemiology, Department of Internal Medicine; and ${ }^{4}$ Division of Vascular Neurology, Department of Neurology University of Utah, Salt Lake City.

OBJECTIVE: Develop an ischemic stroke (IS) knowledge base that accurately predicts IS and its subtypes leveraging predictors from the electronic medical record (EMR). BACKGROUND: In genetic cohort studies, death certificates and hospital-based, ICD-9 billing codes misclassify many non-stroke patients as IS. Predictors in clinical reports may improve classification, but EMR review by physicians can require upwards of 30 minutes per case. To support large-scale genetic study of high-risk pedigrees, we are developing a knowledge base for IS case classification leveraging predictors from ICD-9 codes and clinical reports. DESIGN/METHODS: We are randomly selecting 400 patients hospitalized in the Neurocritical Care Unit at the University of Utah Hospital from 2008 to 2015 with hand-adjudicated discharge diagnoses: 200 IS, 100 hemorrhagic stroke ("HS": intracranial and subarachnoid), and 100 acute neurologic disorders ("Not") to develop the knowledge base and train an IS classifier, and another 200 (100 cases, 100 controls) to test the classifier. We are leveraging a top-down (e.g., domain knowledge from stroke experts) and bottom-up (e.g., predictors from neurologist-confirmed IS cases) modeling approach to develop a computable phenotype of IS predictors documented in the EMR extracted using natural language processing (NLP) and machine learning (ARC/scikit learn) approaches. RESULTS: We developed representations for IS and its subtypes: cardiac embolism, large artery atherosclerosis, lacunar infarction, and cryptogenic including predictors: signs/symptoms ( $\mathrm{n}=11$ types, 515 terms e.g., facial droop), social risk factors ( $\mathrm{n}=1$ type, 105 terms e.g., smoker), findings ( $n=6$ types, 354 terms e.g., stenosis), procedure/interventions ( $n=1$ type, 82 terms e.g., tPA administered), and disease/disorders ( $n=6$ type, 304 terms e.g., ICA stroke). CONCLUSIONS: In conclusion, the knowledge base will support IS case classification reducing time to hand-adjudicate IS cohorts.

Study Supported By: University of Utah Neuroscience Initiative Collaborative Pilot Project Grant.

Disclosure: DLM/WWC: University of Utah BMI \& NI, PCORI CDRN-1306-04912, NLM R00LM011393, NHLBI: R01HL114563 \& R01HL116522, VA HSR\&D: PPO 15-406, RRP 13-420 \& 12-185. BH: none LCA: George E. Wahlen Department of Veterans Affairs Medical Center, Salt Lake City, UT SPLB-001-14S. NIH NCI 1RO1CA195614, 1RO1AG054052, 1R01CA161780-01A1，R01DE023414, DOD PC140624 (PI) W81XWH-14-PCRP-IDA, NIH R01CA164138, U01 CA89600, Huntsman Cancer Foundation JJM: NIH 5U10NS086606, Remedy Pharmaceuticals, Legal consulting for FAVROS PLLC.

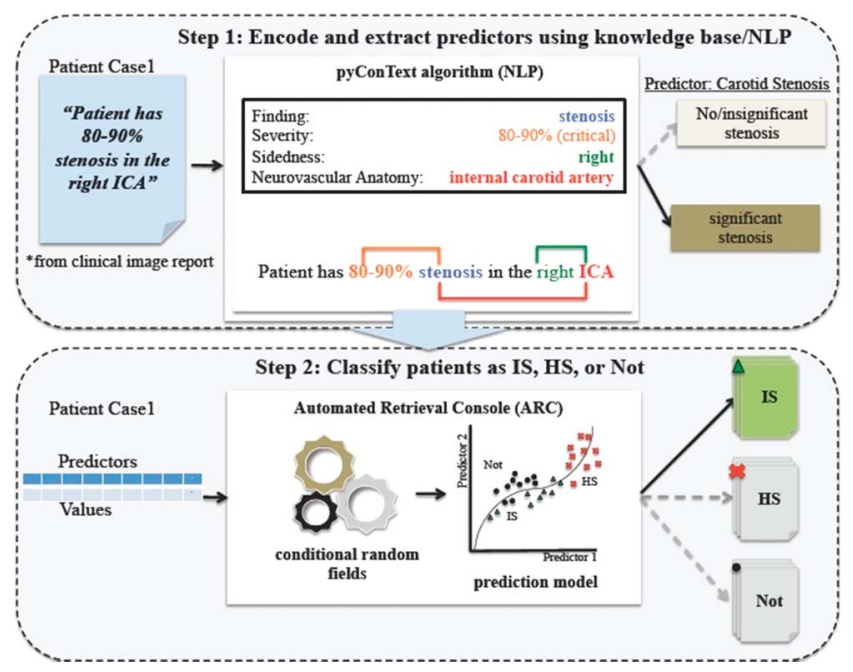

2016.059: Development of a knowledge base to support the automatic classification of a computable ischemic stroke phenotype from electronic medical records
2016.060

An exome array-wide analysis of ischemic stroke in the genetics of early onset stroke study John W. Cole, $M D, M S^{1,2}$, Thomas Jaworek, $B S^{I}$; Kathy Ryan, $M S^{I}$; Patrick McArdle, PhD $D^{I}$; Tushar Dave, PhD $D^{I}$; Oscar C Stine, PhD ${ }^{I}$; Yuching Cheng, PhD ${ }^{I}$; Hugo Aparicio, MD ${ }^{3}$; Qiong Yang, $P h D^{3}$; Hao Mei, $\mathrm{PhD}^{4}$; Megan L. Groves, PhD; ${ }^{5}$ Matthew L. Flaherty, $\mathrm{MD}^{6}$; Steven J. Kittner, $\mathrm{MD}, \mathrm{MPH}^{1,2}$; James

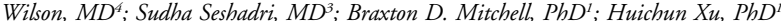

${ }^{1}$ University of Maryland School of Medicine, Baltimore; ${ }^{2}$ Baltimore Veteran's Affairs Medical Center, MD; ${ }^{3}$ Boston University Medical Center, MA; ${ }^{4}$ University of Mississippi Medical Center, Jackson; ${ }^{5}$ University of Texas Health Science Center at Houston; and ' ${ }^{6}$ niversity of Cincinnati, $\mathrm{OH}$.

OBJECTIVE: We sought to identify novel exomic genetic variants that influence stroke risk in young adults. BACKGROUND: Ischemic stroke is a heterogeneous trait and its genetic architecture is poorly understood. The genetic contribution may be stronger for stroke occurring at younger (vs. older) ages. To identify additional stroke susceptibility variants, we genotyped participants in the Genetics of Early Onset Stroke (GEOS) with the exome array and conducted association analyses with the goal of identifying functional risk variants by interrogating the protein-coding portion of genes. DESIGN/ METHODS: The GEOS Study was initiated as a biracial population-based study of cases with first-ever ischemic stroke $15-49$ years of age $(n=828)$ and matched controls $(n=$ 850). All participants underwent exome-chip genotyping. Logistic regression was used to calculate associations in European-Caucasians (EUR) and African-Americans (AA), and summary results were combined by meta-analysis. RESULTS: Our analyses revealed 4 highly associated variants for all-stroke and several others that were stroke subtype specific. Among these for all-stroke, we identified a missense variant in VWDE (rs6460939 $(\mathrm{K}(\mathrm{AAG})->\mathrm{N}(\mathrm{AAC}))$ ), which encodes the Von Willebrand Factor D and EGF domain-containing protein. This protein plays a role in intracellular calcium ion binding within a variety of cell-types. The association was present in both European-Caucasians (OR 1.34, $p=0.002$ ) and African-Americans (OR 1.32, $p=0.01)$, and was strengthened in a meta-analysis of both ethnic groups (OR 1.41, $\left.p=8.95 \times 10^{-6}\right)$. The frequency of the effect $\mathrm{C}$ allele was 0.46 in EUR and 0.55 in AA. Efforts in additional data sets to further replicate this association, and the others identified, are ongoing. CONCLUSIONS: Exome-based analyses in the setting of young-onset stroke is a productive methodology to identify genetic risk variants.

Study Supported By: This study was funded by an American Heart Association CVGPS Pathway Grant (Award 15GPSPG23770000).

Disclosure: None.

\subsection{1}

\section{A genome-wide study of common and rare genetic variants associated with circulating thrombin} activatable fibrinolysis inhibitor (TAFI) Tara M. Stanne, $P h D^{\prime}$, Maja Olsson, $P h D^{1}$. Annie Pedersén, $M D^{1}$. Erik Lorentzen, $M S^{1}$. Anders Gummesson, $M D$, PhD ${ }^{1}$; Ann Gils, PharmD, PhD'; Katarina Jood, $M D, P h D^{3}$; Gunnar Engström $M D, P h D^{4}$, Olle Melander, $M D, P h D^{4}$; Paul J. Declerck, PharmD, PhD'; Christina Jern, $M D, P h D^{\prime}$

${ }^{1}$ Institute of Biomedicine, the Sahlgrenska Academy at the University of Gothenburg, Sweden; ${ }^{2}$ Department of Pharmaceutical and Pharmacological Sciences, KU Leuven, Belgium; ${ }^{3}$ Institute of Neuroscience and Physiology, the Sahlgrenska Academy at the University of Gothenburg, Sweden; and ${ }^{4}$ Department of Clinical Sciences Malmö, Lund University, Sweden.

OBJECTIVE: We sought to identify novel genetic variants that influence plasma concentrations of thrombin-activatable fibrinolysis inhibitor (TAFI) or activated TAFI (TAFIa). BACKGROUND: TAFI plays an important role in regulating endogenous fibrinolysis, where it acts as a functional inhibitor of tissue-type plasminogen activator. Elevated plasma TAFI concentrations have been associated with thrombotic events such as ischemic stroke, myocardial infarction, and deep vein thrombosis. Candidate gene studies have identified common variants that explain only a small fraction of the estimated heritability. DESIGN/METHODS: TAFI and TAFIa were measured in plasma from 3,200 subjects from the case-control Sahlgrenska Academy Study on Ischemic Stroke (SAHLSIS; $\mathrm{n}=600$ cases and $\mathrm{n}=600$ controls) and the prospective Malmö Diet and Cancer study (MDC, $n=2,000$ ). Genotypes from Illumina chips enriched with $240 \mathrm{k}$ exome variants were imputed using the UK10K reference panel (genotyping was performed at the Broad Institute $[\mathrm{n}=2,756]$ and as part of the Stroke Genetics Network [SiGN] study [n = 444]). Genome-wide association scans were conducted to identify genetic variants associated with TAFI or TAFIa. Directly typed rare variants were analyzed using gene-based tests. RESULTS: We confirmed the associations between TAFI concentrations and Thr147Ala (rs3742264) and Thr325Ile (rs1926447) in the gene which encodes TAFI (CPB2; lead SNP TAFI, $p=5.3 \times 10-30$; TAFIa, $\left.p=1 \times 10^{-27}\right)$, and identified a novel genome-wide significant association at 3 p 14.1 for TAFIa $\left(p=1.3 \times 10^{-8}\right)$. Further, we identified 4 novel gene-based associations with TAFIa (all $p \leq 4.3 \times$ $\left.10^{-6}\right)$. CONCLUSIONS: In conclusion, this study identified new candidate genes associated with TAFI and TAFIa for follow-up replication and functional genomic analysis, and provides new information for understanding individual variation in the fibrinolytic pathway. Replicated findings will be tested for association with ischemic stroke.

Study Supported By: This study was supported by the Swedish Heart and Lung Foundation, the Swedish Research Council, the Swedish Stroke Association, and the Sahlgrenska University Hospital. The National Institute of Neurological Disorders and Stroke funded the genotyping of patients included in the SiGN study.

Disclosure: None. 


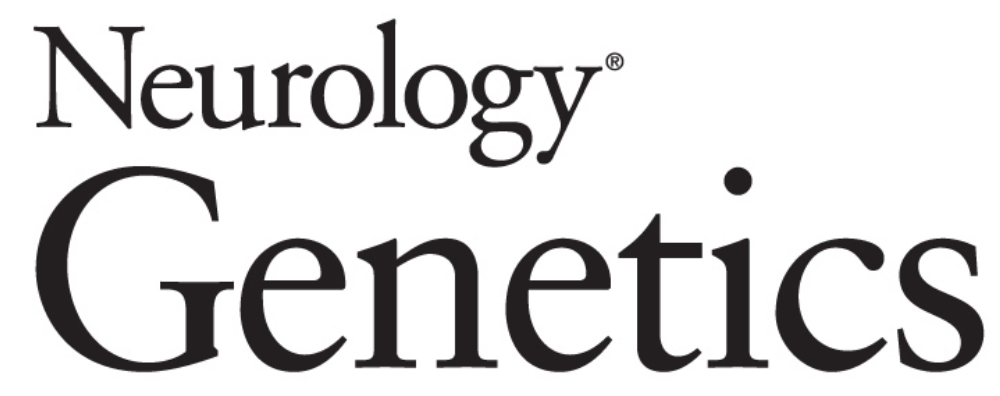
20th Workshop of the International Stroke Genetics Consortium, November 3-4, 2016, Milan, Italy
Neurol Genet 2017;3;S12-S18
DOI 10.1212/NXG.0000000000000136

This information is current as of March 30, 2017

$\begin{array}{ll}\begin{array}{l}\text { Updated Information \& } \\ \text { Services }\end{array} & \begin{array}{l}\text { including high resolution figures, can be found at: } \\ \text { http://ng.neurology.org/content/3/1_Supplement_1/S12.ful. }\end{array} \\ \text { Citations } & \begin{array}{l}\text { This article has been cited by } 1 \text { HighWire-hosted articles: } \\ \text { http://ng.neurology.org/content/3/1_Supplement_1/S12.ful. } \\ \text { rarticles }\end{array} \\ \text { Permissions \& Licensing } & \begin{array}{l}\text { Information about reproducing this article in parts (figures } \\ \text { its entirety can be found online at: } \\ \text { http://ng.neurology.org/misc/about.xhtml\#permissions }\end{array} \\ & \begin{array}{l}\text { Information about ordering reprints can be found online: } \\ \text { http://ng.neurology.org/misc/addir.xhtml\#reprintsus }\end{array}\end{array}$

Updated Information \&

Citations

Permissions \& Licensing

Reprints including high resolution figures, can be found at:

http://ng.neurology.org/content/3/1_Supplement_1/S12.full.html

This article has been cited by 1 HighWire-hosted articles:

http://ng.neurology.org/content/3/1_Supplement_1/S12.full.html\#\#othe articles

(tables) or in its entirety can be found online at:

Information about ordering reprints can be found online:

http://ng.neurology.org/misc/addir.xhtml\#reprintsus

Neurol Genet is an official journal of the American Academy of Neurology. Published since April 2015, it is an open-access, online-only, continuous publication journal. Copyright (C) 2017 American Academy of Neurology. All rights reserved. Online ISSN: 2376-7839.

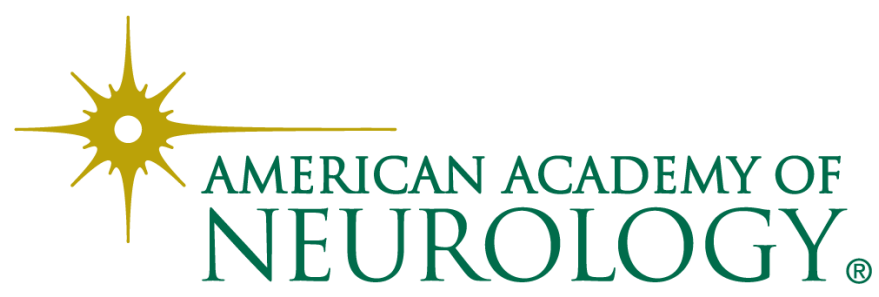

